\title{
Latente Hyperthyreose als kardiales Risiko
}

\section{Die endogene latente Hyperthyreo- se geht mit einem erhöhten Risiko einer kardiovaskulären Mortalität und für Vorhofflimmern einher, das graduell mit der Suppression des TSH zunimmt.}

- Eine mögliche Assoziation zwischen einer latenten Hyperthyreose und kardiovaskulären Erkrankungen wurde in mehreren prospektiven Kohortenstudien untersucht, die Ergebnisse sind jedoch widersprüchlich. Die gepoolten Daten von insgesamt fast 52700 Studienteilnehmern aus insgesamt zehn Kohorten wurden nun auf einen möglichen Zusammenhang zwischen Hyperthyreose und koronarer Herzkrankheit untersucht.

Bei 22400 Teilnehmern aus sechs Kohorten war es zu koronaren Ereignissen gekommen, bei 8711 Teilnehmern aus fünf Kohorten war Vorhofflimmern aufgetreten. Die Euthyreose in diesen Studien war definiert durch einen TSHWert zwischen 0,45 und $4,49 \mathrm{mIU} / \mathrm{l}$ bei normalem freien Thyroxin. Von den über 52000 Teilnehmern hatten 2188 (4,2 \%) eine latente Hyperthyreose.

Im Follow-up verstarben 8527 Personen, 3653 von 22437 erlitten koronare Ereignisse und 785 von 8711 entwi-

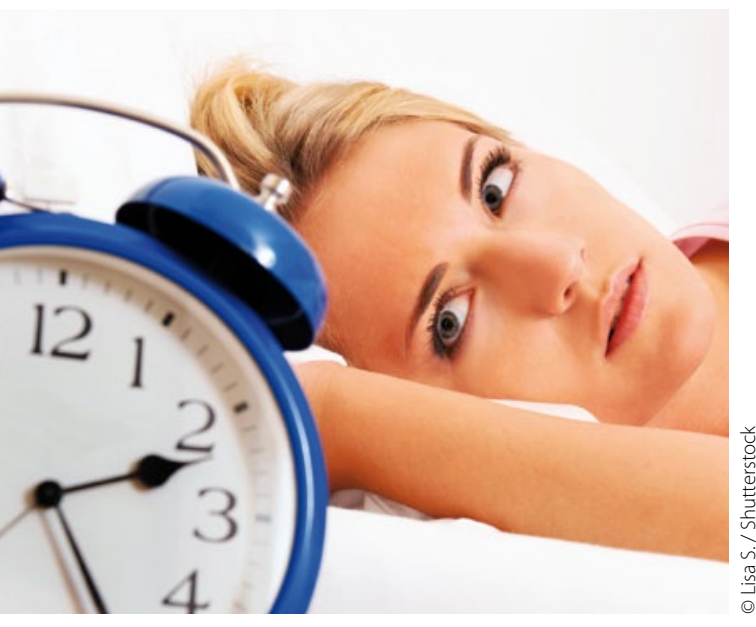

Zu kurzer Schlaf lässt den Stoffwechsel entgleisen. ckelten Vorhofflimmern. In einer altersund geschlechtsadjustierten multivariaten Analyse war die latente Hyperthyreose mit einer erhöhten Gesamtmortalität (HR 1,24), einer koronaren Mortalität (HR 1,29), koronaren Ereignissen (HR 1,21) und Vorhofflimmern (HR 1,68) assoziiert. Das für die latente Hyperthyreose attributable Risiko lag bei $14,5 \%$ für die Gesamtmortalität und bei 41,5\% für Vorhofflimmern. Risiken für die koronare Mortalität und Vorhofflimmern zeigten einen dosisabhängigen Effekt und waren für TSH-Spiegel $<0,1 \mathrm{mIU} / \mathrm{l}$ höher als für TSH-Konzentrationen zwischen 0,1 und $0,44 \mathrm{mIU} / \mathrm{l}$.

\footnotetext{
-T. H. Collet et al.

(Korres.: Nicholas Rodondi, MD, Department of General Internal Medicine, Inselspital, University of Bern, 3010 Bern; nicholas.rodondi@insel. ch): Subclinical hyperthyroidism and the risk of coronary heart disease and mortality. Arch. Intern. Med. 2012; published online doi:10:1001/ archinternmed. 2012.402
}

\section{Kommentar}

Die Frage einer möglichen Assoziation zwischen latenter Hyperthyreose, koronaren Ereignissen und vor allem Vorhofflimmern scheint somit aufgrund der Analyse dieser Daten entschieden. Allerdings gelingt es mit Kohortenstudien grundsätzlich nicht, Kausalitäten zu belegen. Noch viel weniger erlauben uns die Daten eine fundierte Entscheidung, ob Patienten mit latenter Hyperthyreose thyreostatisch behandelt werden sollen oder nicht. Diese Frage lässt sich nicht mit einer relativ bequem am Computer durchführbaren Metaanalyse beantworten, sondern bedarf einer prospektiven, randomisierten und kontrollierten Studie. Bei der geringen Effektstärke, die von einer Therapie zu erwarten ist, wären für eine verlässliche Aussage allerdings sehr große Patientenzahlen erforderlich, so dass eine derartige Studie wohl nicht zustande kommen wird.

H. S. FÜESSL :

\section{Kurzschläfer werden dicker}

\section{Der Körper benötigt Ruhe, um Fett und Zucker zu verarbeiten. Fehlt der Schlaf, gerät der Stoffwechsel durcheinander. Die Fähigkeit der Fettzellen, auf das Hormon Insulin zu reagieren, wird bei zu kurzem Schlaf deutlich reduziert, wie US-Forscher berichten.}

- Die Forscher untersuchten sechs Frauen und einen Mann. Alle Probanden waren jung, schlank und gesund. In einem Studienabschnitt mussten sie vier Nächte hintereinander jeweils 8,5 Stunden im Bett verbringen. In dem zweiten Abschnitt durften sie dort nur 4,5 Stunden liegen. In beiden Fällen erhielten sie die gleichen Lebensmittel.

Nach den vier Nächten mit Schlafmangel reagierte der Körper um 16\% weniger gut auf Insulin. Die Empfindlichkeit der in der Nabelgegend entnommenen Fettzellen für Insulin sank sogar um 30\% $(\mathrm{P}=0,01)$. Eine solche Reduktion finde sich auch bei Übergewichtigen und bei Diabetikern, schreiben die Forscher.

\section{Kommentar}

Die Studie ist klein, aber gründlich. Alle sieben Probanden haben eine deutliche Änderung in der Empfindlichkeit für Insulin gezeigt. Schlafrestriktion führt zur Insulinresistenz der humanen Fettzellen. Schlaf kann ein bedeutender Regulator des Energiestoffwechsels der peripheren Gewebe sein.

K. MALBERG =

- J. L. Boussard et al.

Autorenkorrespondenz: MJ Brady, The University of Chicago, KCBD 8124, 900 East 57th St, Chicago, III. 1637 USA; E-mail: mbrady@medicine.bsd.uchicago) Impaired insulin signaling in human adipocytes after experimental sleep restriction: A randomized, crossover study. Ann. Int. Med. 16 Oct 2012: 157 (8) 540-547 